\title{
ACTUALIZACIÓN DEL TRATAMIENTO DE LA POLINEUROPATÍA DIABÉTICA PERIFÉRICA DOLOROSA 2015. COMITÉ DE NEUROPATÍA DIABÉTICA. SOCIEDAD ARGENTINA DE DIABETES
}

\author{
TREATMENT UPDATE OF PAINFUL PERIPHERAL DIABETIC \\ NEUROPATHY. DIABETIC NEUROPATHY COMMITTE 2015. \\ ARGENTINE SOCIETY OF DIABETES
}

Carolina Domínguez', Claudia Flores², Graciela Fuente, Concepción García', Marcela Giménez Rey³, Solange Houssay ${ }^{4}$, Fernanda Huber ${ }^{1}$, Carlos Santillán ${ }^{5}$, Raquel Urdaneta ${ }^{1}$

\section{RESUMEN}

La diabetes es una enfermedad crónica y progresiva que se asocia a complicaciones potencialmente discapacitantes, entre ellas, la neuropatía diabética es probablemente la afectación sintomática más frecuente con importante impacto social y económico. El tratamiento de la neuropatía somática dolorosa y del sistema nervioso autónomo ha sido un desafío durante años. Nuevas terapéuticas se encuentran disponibles, las cuales mejoran el dolor y brindan esperanza de lograr alivio a personas que padecen esta complicación. Palabras claves: neuropatía diabética, hiperalgesia, tratamiento
patogénico, tratamiento sintomático.

Revista de la Sociedad Argentina de Diabetes 2016; Vol. 50 (35-46)

\section{ABSTRACT}

Diabetes is a chronic and progressive disease associated with potentially disabling complications. Among them, diabetic neuropathy is probably the most frequent symptomatic manifestation, having an important social and economic impact. Painful somatic neuropathy and autonomic neuropathy treatment have been a challenge for years. New medical approaches that relief pain are now available and give hope to diabetic patients.

Key words: diabetic neuropathy, hyperalgesia, pathogenic treatment, symptomatic treatment.

Revista de la Sociedad Argentina de Diabetes 2016; Vol. 50 (35-46)
Hospital Carlos G. Durand, CABA, Argentina

2 Hospital Dr. José Penna, Bahía Blanca, Prov. de Bs. As., Argentina

3 Hospital Gbdor D. Mercante, José C. Paz, Prov. de Bs. As., Argentina

4 Hospital Gral. Agudos E. Tornú, CABA, Argentina

5 Hospital Dr. Leónicas Lucero, Bahía Blanca, Prov. de Bs. As., Argentina
Contacto del autor: Raquel Urdaneta Vélez

E-mail: raquelurdaneta@live.com.ar

Correspondencia: Servicio de Nutrición Hospital.

Av. Díaz Vélez 5044 (C1405DCS), CABA, Argentina

Tel.: (011) 1165483300

Fecha de trabajo recibido: 19/02/16

Fecha de trabajo aceptado: 12/04/16

Conflicto de intereses: los autores declaran no tener conflictos de interés.

\section{INTRODUCCIÓN}

La neuropatía periférica es una complicación común de la diabetes mellitus, que se presenta entre el 30 y el $50 \%$ de los pacientes. La neuropatía puede manifestarse con la pérdida de la sensibilidad en forma simétrica, a modo de media o guante, comenzando en los dedos de los pies y progresando en forma proximal. Aproximadamente del $10 \mathrm{al}$ $20 \%$ de los pacientes con diabetes tienen neuropatía periférica dolorosa, cuyos síntomas son dolor tipo quemante, lacerante o punzante y que empeora en horas de la noche. El dolor también puede presentarse en forma de alodinia o hiperalgesia.

La polineuropatía diabética periférica (PNDP) dolorosa interfiere en la calidad del sueño, el humor y en las actividades diarias del paciente.

El manejo inicial de esta complicación incluye el control de la hiperglucemia, teniendo en cuenta que la optimización glucémica podría empeorar el dolor (neuritis insulínica)'. 
Estudios epidemiológicos han sugerido que los pacientes con pobre control glucémico tienen mayor riesgo de desarrollar neuropatía. Un aumento en la hemoglobina glicosilada A1c del $2 \%$ conlleva un incremento del riesgo de padecer esta complicación de 1,6 veces en un período de 4 años $^{2}$. Otros estudios como el DCCT y el UKPDS concluyeron que el control glucémico intensivo enlentece el desarrollo y/o la progresión de la neuropatía $3,4,5$.

Las metas de tratamiento incluyen restaurar la función nerviosa y mejorar el dolor, sin embargo, con frecuencia los pacientes sólo pueden esperar la reducción real del dolor entre el 30 al 50\%, con mejoría funcional.

Las principales drogas usadas en el tratamiento de PNDP dolorosa incluyen: antidepresivos tricíclicos, anticonvulsivantes, inhibidores de la recaptación de serotonina y noradrenalina, opioides y sustancias opioides-like y medicación tópica. Actualmente existen terapéuticas alternativas o complementarias que podrían ser de utilidad en el tratamiento del dolor. Lamentablemente la evidencia disponible en el tratamiento de la PNDP dolorosa está limitada a estudios pequeños y muy pocos de éstos son comparativos entre drogas (head-tohead). Además como estos pacientes presentan en general múltiples comorbilidades, los médicos deben considerar los potenciales efectos adversos y las posibles interacciones medicamentosas antes de prescribir una medicación ${ }^{6}$.

\section{Mecanismos de injuria tisular que conducen al desarrollo de complicaciones crónicas de la diabetes}

Las bases fisiopatológicas de la PNDP incluyen los efectos metabólicos de la hiperglucemia como la generación de potenciales neurotoxinas (especies reactivas del oxígeno y el sorbitol) y los cambios en el nivel intracelular de enzimas y otras señales moleculares ${ }^{7,8}$ (Gráfico 1).

Los mecanismos involucrados en el desarrollo de la neuropatía diabética (ND) son la activación de la vía de los polioles, activación de la proteína kinasa $C$, el estrés oxidativo, la activación de la poli (ADP-ribosa) polimerasa, la alteración de factores neurotróficos, la formación de AGEs, las anormalidades en ácidos grasos esenciales y los mecanismos inmunológicos.

Esta base fisiopatológica es la que da sustento a las opciones terapéuticas disponibles.

Una vez diagnosticada la PNDP existen dos enfoques terapéuticos: el patogénico, cuyo blanco es la prevención del proceso de pérdida de fibras nerviosas, y el sintomático, cuyo principal objetivo es aliviar los síntomas de dolor para optimizar el funcionamiento físico y el bienestar psicológico.

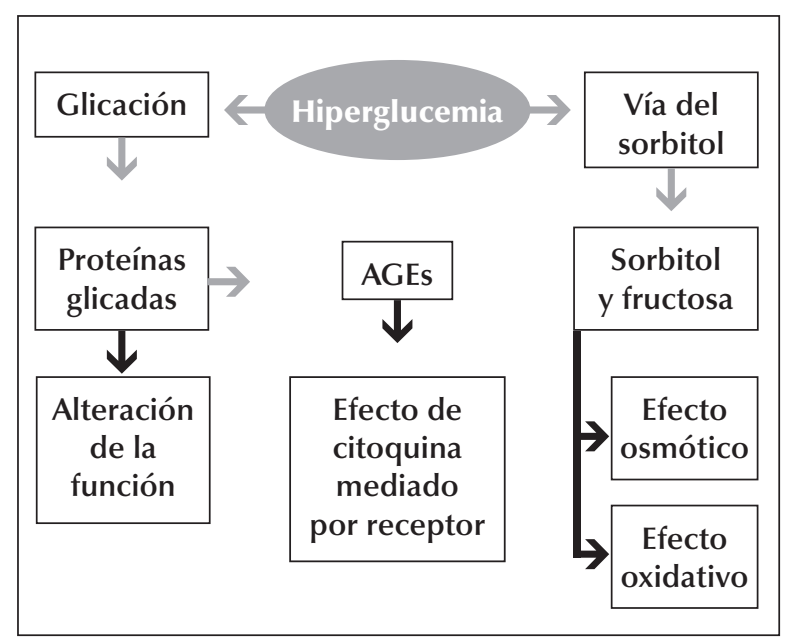

Gráfico 1: Efectos metabólicos de la hiperglucemia.

\section{TRATAMIENTO}

Con un criterio didáctico se puede clasificar el tratamiento en patogénico y sintomático y el sintomático a su vez, en enfoque farmacológico y no farmacológico.

\section{Tratamiento patogénico}

- Control glucémico

La primera medida a considerar es el control glucémico, como fue demostrado en distintos estudios multicéntricos, entre los cuales el DCCT evidenció que la prevalencia de ND se mantenía baja en la rama de tratamiento intensificado, enfatizando el papel de la insulina en la prevención tanto primaria como secundaria de la neuropatía en pacientes con diabetes mellitus tipo $1(\mathrm{DM} 1)^{3}$. La prevalencia de la polineuropatía distal permaneció más baja en el grupo intensificado (22 vs $28 \%$ ), incluso varios años después de iniciada la intervención, lo que se denominó "memoria metabólica"10,11.

En los pacientes con DM tipo 2 (DM2) el control intensificado en prevención y manejo de la ND es menos claro, encontrándose conclusiones contradictorias. EI UKPDS mostró una disminución en el índice de empeoramiento del umbral de percepción vibratoria (UPV) después de 15 años de terapéutica intensiva versus convencional (31 vs $52 \%)^{5}$. En el estudio ADVANCE no se obtuvo 
diferencia significativa luego de un seguimiento medio de cinco años entre ambos grupos ${ }^{12}$. En el VADT luego de un seguimiento de cinco a seis años, no hubo diferencias significativas ${ }^{13}$. Un reciente metaanálisis encontró que no existe reducción de la incidencia de la ND en pacientes con $\mathrm{DM} 2$, con el control glucémico intensivo ${ }^{14}$.

Estas conclusiones también están avaladas en una revisión Cochrane en la cual se demuestra que no existe mejoría significativa en los marcadores de ND en estos pacientes bajo tratamiento intensificado ${ }^{15}$. De todos modos hay que considerar que varios de estos estudios no fueron diseñados para evaluar los efectos del control intensificado sobre ND.

El uso de las bombas de insulina asegura la infusión continua de insulina subcutánea y se utiliza en especial en el tratamiento intensificado. Permiten obtener un perfil de insulina similar al fisiológico, aunque no están exentas de riesgo. Los pacientes con ND hiperalgésica pueden beneficiarse con su uso ${ }^{16,17}$.

El trasplante de páncreas es la única terapia reconocida que restaura la secreción endógena de insulina en respuesta a mecanismos de retroalimentación. Se ha sugerido que la reversión de la ND tomaría 10 años después del trasplante ${ }^{18}$. Los resultados de este procedimiento están limitados por la disponibilidad de órganos, las complicaciones de la cirugía y las consecuencias de la inmunosupresión.

\section{- Ácido alfa lipoico o tióctico}

Uno de los mecanismos involucrados en el desarrollo de la ND es el estrés oxidativo, en el cual el aumento de la producción de radicales libres junto con mecanismos antioxidantes defectuosos sería uno de los responsables del desarrollo de ND ${ }^{19}$.

El ácido alfa lipoico (ALA) ha sido empleado por sus propiedades antioxidantes, mostrando su influencia favorable sobre la microcirculación y la mejoría de los síntomas neuropáticos 20,21,22,23.

En el estudio SYDNEY 2 se sugiere que el tratamiento con ALA 600 mg/día por vía oral durante cinco semanas reduce los principales síntomas de ND, incluyendo dolor, parestesias y adormecimiento en un grado clínicamente significativo ${ }^{24}$. Un estudio más reciente (NATHAN 1) donde se incluyeron 460 pacientes diabéticos con estadios 1 ó 2 de ND fueron randomizados a ALA 600 mg/ una vez por día $(n=233)$ o placebo $(n=227)$ por cuatro años. Después de este período el Neuropathy Impairment Score (NIS) se mejoró, pero no así la velocidad de conducción nerviosa (VCN); la droga fue bien tolerada durante todo el estudio ${ }^{25}$.

El ALA ha sido aprobado en varios países para ambos enfoques terapéuticos.

Las vías de administración disponibles son oral y endovenosa, ambas bien toleradas. El efecto analgésico se evidencia más tempranamente con la infusión endovenosa, teniendo la precaución de utilizar vías con fotoprotección debido a su fotosensibilidad. Un metaanálisis de cuatro estudios controlados con placebo realizado por Ziegler et al., describió una mejoría significativa en los síntomas de ND después de la administración de ALA $600 \mathrm{mg} /$ día durante tres semanas ${ }^{26}$.

Los efectos adversos que se han informado fueron náuseas y malestar digestivo. Por su efecto insulinosensibilizador debe prestarse atención a la aparición de hipoglucemia.

El rango de dosis para la vía oral es de 600 a $1.800 \mathrm{mg} / \mathrm{d}$. Su absorción se ve afectada por los alimentos por lo cual se aconseja su administración alejada de las comidas. En el caso de no poder utilizar la dosis de 1.800 mg/d, la administración de 600 mg/d logra mejoría sintomática a las cinco semanas de iniciado el tratamiento.

En casos severos o de intolerancia digestiva, el ALA puede emplearse por vía endovenosa; en la actualidad en nuestro medio se encuentra disponible en ampollas de $600 \mathrm{mg}$.

\section{- Actovegin}

El actovegin es un producto hemoderivado desproteinizado por ultrafiltración de sangre de ternero que contiene componentes de bajo peso molecular hasta 5.000 Dalton. El actovegin estimula la absorción y utilización de oxígeno, así como también el metabolismo energético celular ${ }^{27}$. En las ratas diabéticas por estreptozotocina (STZ) hay una mejoría significativa en la velocidad de conducción nerviosa sensitiva y en la densidad de fibras nerviosas intraepidérmicas (DFNDI) luego del tratamiento con actovegin; más aún, este componente descendió marcadamente la apoptosis celular del nervio ciático del mismo tipo de ratas, medida por el contenido de la poly-ADP-ribosa ${ }^{28}$.

La eficacia y la seguridad del actovegin en ND fueron evaluadas en un estudio multicéntrico, randomizado, doble ciego que incluyó 567 pacientes con DM2, que recibieron 20 infusiones intravenosas de actovegin ( $2.000 \mathrm{mg} /$ día) o placebo, una vez por día, seguidas de tres tabletas de actovegin $(1.800 \mathrm{mg} / \mathrm{d})$ 
o placebo, tres veces por día durante 140 días. No hubo diferencias estadísticamente significativas ${ }^{27}$.

En el momento de esta revisión no se cuenta con este fármaco en nuestro país.

\section{- Benfotiamina}

Es un análogo liposoluble de la tiamina (vitamina B1) que ha demostrado en modelos animales inhibir las tres vías metabólicas involucradas en el daño microvascular asociado a diabetes: vía de las hexosaminas, de la formación de los productos avanzados de glicación no enzimática y la vía de la proteína kinasa $C$, a través de la transcetolasa ${ }^{29}$. En el estudio BENDIP (Benfotiamin in Diabetes Polyneuropathy), ensayo clínico randomizado doble ciego, controlado con placebo se evaluaron la eficacia y tolerancia de dos dosis diferentes de esta droga, 300 y $600 \mathrm{mg}$ por día durante seis semanas. El resultado primario del estudio fue una mejoría significativa del Neuropathy Symptom Score (NSS) mediante el análisis PP (per protocol), mientras que en el análisis de intención a tratar (intention to treat, ITT) el resultado fue apenas por encima del nivel de significancia. De los síntomas evaluados el resultado más relevante fue la mejoría del dolor mientras que las parestesias lo hicieron con menor frecuencia. En el estudio BEDIP (Benfotiamine in Diabetic Polyneuropathy), investigación piloto randomizada controlada con placebo, la benfotiamina mejoró el score NSS. En ambos estudios (BENDIP y BEDIP) los resultados no se atribuyeron a cambios metabólicos dado que no hubo diferencias en niveles de $\mathrm{HbA} 1 \mathrm{c}$ ni en el perfil glucémico entre los grupos ${ }^{30}$.

- Inhibidores de la aldosa reductasa

Los inhibidores de la aldosa reductasa (IAR) actúan sobre la vía metabólica de los polioles previniendo la reducción de los potenciales de óxidoreducción. El análisis de los estudios clínicos aleatorizados controlados con placebo evidenció que el tolrestat contribuyó a la mejoría de la sensibilidad vibratoria en ND. La prescripción de zenarestat por 12 meses se acompañó con cambios, dosis-dependientes en la densidad del tejido nervioso, aumentando la velocidad de los impulsos nerviosos. Zopolrestat y ranirestat son una nueva generación de IAR que muestran eficacia en estudios experimentales.

Un gran número de IAR ha demostrado efectos adversos severos que motivaron que no estuvieran en el mercado farmacéutico o que fueran retirados del mismo. Se han observado reacciones de hipersensibilidad severas (sorbinil), aumento de la creatinina (zenalrestat) e injuria hepática (tolrestat) ${ }^{31}$.

El IAR epalrestat ha mejorado los síntomas de ND reportados por los pacientes con un aceptable perfil de seguridad; un estudio aleatorizado a tres años sugirió un rol preventivo para la neuropatía diabética distal y simétrica (NDDS) ${ }^{32,33}$.

En el momento de esta revisión no se cuenta con este fármaco en nuestro país.

\section{- Ácido gama linolénico y L-carnitina}

Existen distintos estudios en los que se han utilizado ácido gama linolénico y L-carnitina en neuropatía periférica, encontrándose en alguno de ellos mejoría electrofisiológica, sin embargo no son drogas de uso habitual en nuestro medio $0^{34,35,36}$.

\section{Tratamiento sintomático}

Para el tratamiento del dolor neuropático existen terapias farmacológicas de uso sistémico y local, y terapias no farmacológicas.

\section{Tratamiento farmacológico}

Dentro del tratamiento farmacológico se encuentran los antidepresivos, anticonvulsivantes, antagonistas del receptor n-metil-d-aspartato (NMDA), analgésicos opioides y opioides atípicos y agentes tópicos.

En el Gráfico 2 se puede observar el sitio de acción de las diferentes drogas, en la Tabla 1 los niveles de evidencia y en la Tabla 2 las dosis utilizadas.

\section{- Drogas antidepresivas}

Las drogas antidepresivas incluyen a los antidepresivos tricíclicos, los inhibidores selectivos de la recaptación de serotonina y los inhibidores de la recaptación de serotonina y noradrenalina.

- Antidepresivos tricíclicos: son los agentes de primera línea para el manejo del dolor neuropático ${ }^{37}$.

- Amitriptilina: la amina terciaria amitriptilina es el agente más estudiado dentro de los antidepresivos tricíclicos y ha demostrado en numerosos estudios randomizados, controlados con placebo, que mejora significativamente el dolor neuropático. Estos agentes inhiben la recaptación de noradrenalina y serotonina, y también pueden bloquear los canales de sodio voltaje dependiente. Además antagonizan los receptores n-metil-daspartato (NMDA) que median la hiperalgesia y la alodinia $^{38,39}$. La acción central de estos fármacos 
acentúa el efecto de los neurotransmisores en la activación de sistemas inhibitorios del dolor a nivel cerebral que a su vez modulan la transmisión del dolor en células de la médula espinal ${ }^{38}$.

Los efectos adversos más frecuentes de los antidepresivos tricíclicos son cansancio y boca seca. La amitriptilina tiene efecto sobre la función tiroidea por lo cual la misma debe ser evaluada ${ }^{40}$. Existe riesgo de síndrome serotoninérgico si se suma a otro antidepresivo o al tramado| ${ }^{41}$.

La amitriptilina comparada con gabapentina muestra igual eficacia analgésica ${ }^{42,43,44}$.

La dosis de inicio es de $25 \mathrm{mg}$, tomada una vez al día, una hora antes de dormir. La misma puede incrementarse con intervalos semanales de a $25 \mathrm{mg}$ hasta aliviar el dolor, o hasta que aparezcan efectos adversos. La dosis máxima es de 150 mg/día.

La analgesia con amitriptilina es similar en subgrupos con depresión y sin depresión, y la mejoría del dolor no se relaciona con la mejoría del humor ${ }^{45}$.

Estos fármacos deben usarse con cuidado en pacientes con hipotensión ortostática y están contraindicados en pacientes con angina inestable, infarto de miocardio reciente (menos de seis meses), insuficiencia cardíaca, arritmias ventriculares y síndrome del QT largo. Se sugiere realizar un electrocardiograma a todo paciente con ND mayor de 40 años antes de indicar esta medicación ${ }^{46,47}$.

- Inhibidores selectivos de la recaptación de serotonina (ISRS): Ios ISRS inhiben la recaptación presináptica de serotonina. En este grupo se encuentran la fluoxetina, paroxetina, citalopram y escitalopram que poseen un efecto débil sobre el dolor neuropático ${ }^{48}$.

- Inhibidores de la recaptación de serotonina y noradrenalina (IRSN): en el grupo de IRSN se incluyen la duloxetina y venlafaxina.

. Duloxetina: la eficacia y seguridad de la duloxetina fue evaluada en distintos estudios empleando dosis de 60 y 120 mg/día, resultando ambas dosis efectivas en el manejo del dolor ${ }^{49}$. Un trabajo realizado con duloxetina con $60 \mathrm{mg}$ y $120 \mathrm{mg}$ contra placebo demostró una mejoría concreta en la severidad en la escala de dolor en 24 hs; todas las mediciones mejoraron excepto la alodinia ${ }^{50}$.

En un trabajo realizado en el que se comparó monoterapia con duloxetina versus duloxetina/gabapentina no se observó superioridad en la combinación de drogas ${ }^{51}$. La duloxetina no debe utilizarse en insuficiencia hepática y con clearence de creatinina inferior a $30 \mathrm{ml} /$ minuto $^{52}$.
Los efectos adversos más frecuentes incluyen: somnolencia, náuseas, constipación y mareos, que pueden reducirse iniciando el tratamiento con una dosis de $30 \mathrm{mg} /$ día durante cuatro a cinco días ${ }^{53}$.

Venlafaxina: a dosis bajas inhibe la recaptación de serotonina y a dosis más altas inhibe la recaptación de noradrenalina ${ }^{47,54}$. Si bien está aprobada para desórdenes depresivos mayores, ansiedad generalizada, desorden de ansiedad social y desorden del pánico, no está aprobada por la Administración Federal de Drogas y Alimentos de Estados Unidos (FDA, sus siglas en inglés) para el tratamiento del dolor neuropático ${ }^{55}$.

\section{- Drogas antiepilépticas}

Dentro de las drogas antiepilépticas o anticonvulsivantes se incluyen los moduladores de canales de calcio y los bloqueantes de canales de sodio.

- Moduladores de los canales de calcio.

Pregabalina: fue el primer anticonvulsivante aprobado por la FDA para el tratamiento de la neuralgia post-herpética, la polineuropatía diabética hiperalgésica y el dolor neuropático secundario a la lesión de la médula espinal.

Es un análogo del ácido gamma-aminobutírico (GABA), que se une en forma selectiva a la subunidad $\alpha 2 \delta$ de los canales de calcio dependientes del voltaje de las terminaciones presinápticas del cerebro y de la médula espinal, inhibiendo la liberación de neurotrasmisores excitatorios ${ }^{56}$.

En varios estudios clínicos se demostró la eficacia de la pregabalina en el control del dolor neuropático, siendo administrada en forma progresiva en dosis de 300 a 600 mg/día con un NNT para aliviar en al menos un 50\% el dolor en una de 6,3 personas ${ }^{57,58}$. Se sugiere comenzar con una dosis de inicio de 25 a $150 \mathrm{mg} /$ día con aumentos semanales de 25 a $100 \mathrm{mg} / \mathrm{día}^{59}$. Además de sus efectos analgésicos, la pregabalina presenta un efecto ansiolítico, mejora el sueño y la calidad de vida, contribuyendo a mejorar las condiciones generales de los pacientes.

Los efectos adversos asociados al tratamiento incluyen: mareos, somnolencia, edemas periféricos, visión borrosa, cefalea y ganancia de peso ${ }^{60}$.

Gabapentina: es también un anticonvulsivante relacionado estructuralmente al GABA, un neurotransmisor que tiene un rol preponderante en la trasmisión y la modulación del dolor. Presenta gran afinidad por la subunidad $\alpha 2 \delta$ de los canales 
de calcio activados por voltaje. En un ensayo clínico multicéntrico de ocho semanas de titulación de dosis, con 165 pacientes con neuropatía diabética hiperalgésica, el $60 \%$ de los pacientes tratados con gabapentina ( $3.600 \mathrm{mg} / \mathrm{día}$ ) logró una reducción de al menos el $50 \%$ en el dolor, comparado con el $33 \%$ del grupo placebo. Los mareos y la somnolencia fueron los efectos adversos más frecuentes y ocurrieron en $23 \%$ de los pacientes ${ }^{61}$. El NNT para la reducción del dolor neuropático con gabapentina es de 6,4. Mejora el sueño, comprometido con mucha frecuencia en la neuropatía dolorosa crónica y a largo plazo produce ganancia de peso que complicaría el tratamiento de la diabetes y sus comorbilidades asociadas ${ }^{62}$. La combinación con opiáceos le suma eficacia analgésica ${ }^{63}$.

La pregabalina y gabapentina tienen un mecanismo de acción similar y algunos ensayos clínicos orientan a mostrar una eficacia analgésica superior a los antidepresivos tricíclicos o a los opiáceos. Otros aspectos importantes de estas drogas incluyen su buena tolerancia y la ausencia de efectos adversos graves ${ }^{64}$.

- Bloqueantes de los canales de sodio

En las zonas de injuria nerviosa se acumulan canales de sodio que generan hiperexcitabilidad y descargas espontáneas tanto en el sitio de la injuria como en las neuronas de las raíces dorsales produciendo como consecuencia dolor quemante o ardor ${ }^{65}$.

Carbamazepina, oxcarbazepina, lacosamida: si bien la carbamazepina ha sido ampliamente utilizada para tratar el dolor neuropático, no puede ser recomendada para el tratamiento de la neuropatía dolorosa en base a las evidencias limitadas en cuanto a su eficacia y seguridad. Su sucesora, la oxcarbazepina, así como otros bloqueantes de canales de sodio como valproato, mexiletina, topiramato, lamotrigina y lacosamida mostraron eficacia marginal y no han obtenido aún la aprobación de las entidades regulatorias para ser utilizadas en el tratamiento de la neuropatía diabética hiperalgésica ${ }^{59,66}$.

\section{- Opioides y opioides-like}

Dentro de los opioides se incluyen a la metadona, levorfanol, morfina, oxicodona y dentro de los opioides-like, el dextrometorfano, tapentadol y tramadol. Los utilizados en nuestro medio son la morfina, oxicodona, dextrometorfano, tapentadol y tramadol.

Morfina: existen estudios que muestran be- neficios sólo en una porción de pacientes que logró una reducción modesta del dolor en un 20 a un $30 \%$ y fue evaluada en unas pocas semanas ${ }^{67}$.

La dosis utilizada es entre 15 a 120 mg/día.

Los eventos adversos y su potencial adicción limitan su uso. Puede utilizarse combinada con gabapentina en dosis menores, obteniendo iguales efectos analgésicos ${ }^{63,68}$.

Oxicodona: no hay mucha evidencia disponible, pero puede utilizarse en combinación con otras drogas ${ }^{63,68}$.

Dextrometorfano (dextromethorphan sh15): es un antagonista del receptor glutaminérgico nmetil-d-aspartato (NMDA). Los receptores NMDA juegan un importante rol en la sensibilización central del dolor neuropático. Su uso no ha sido muy difundido debido a los efectos adversos que limitan su dosis 69 .

.Tapentadol: es un analgésico central con modo de acción dual, agonista del receptor $\mu$-opioide e inhibidor de la recaptación de noradrenalina. En estudios recientes de fase III se evaluaron la seguridad y eficacia de tapentadol de liberación prolongada en polineuropatía distal y simétrica en pacientes diabéticos, logrando una reducción del dolor comparado con placebo. La dosis utilizada es de 50-250 mg, en dos tomas diarias ${ }^{70}$.

. Tramadol: analgésico opioide débil de acción central, que se emplea para el manejo del dolor moderado a severo. Actúa directamente en receptores opioides e indirectamente por el sistema de receptores monoaminérgicos. No es frecuente el desarrollo de tolerancia a esta droga ${ }^{71}$.

Para el uso de opioides y opioides-like hay que tener en cuenta sus efectos adversos y la potencial adicción que pueden originar. No hay datos suficientes para recomendar un agente $u$ otro, y están contraindicados con el uso de los IMAO.

\section{- Agentes tópicos}

Dentro del tratamiento local se incluyen la capsaicina tópica, lidocaína, amitriptilina y toxina botulínica.

. Capsaicina tópica: la capsaicina es un alcaloide derivado del ají, que se liga al receptor transient receptor potential V1 (TRPV1) activando los canales de calcio y generado su apertura. La activación prolongada del receptor produce una disminución de neurotransmitores, en particular de la sustancia $\mathrm{P}$, reduciendo así la capacidad de las neuronas para transmitir señales de dolor ${ }^{72}$.

La crema de capsaicina tiene presentación en 
concentraciones al 0,025-0,25\% y se puede aplicar varias veces en el día durante varias semanas. Existe una presentación en parche al 8\%, el cual se aplica luego de analgesia local con lidocaína tópica produciendo alivio del dolor durante tres meses. Entre los efectos adversos locales se destacan eritema, ardor y dolor localizado en el sitio de aplicación ${ }^{73,74}$.

Las Guías NICE recomiendan el uso de crema de capsaicina en pacientes con neuropatía localizada. La Asociación Americana de Neurología (AAN) la recomienda para el tratamiento de NPD (nivel B) $)^{75}$. En el año 2009 la FDA aprobó el parche de capsaicina $8 \%(\text { Qutenza })^{73}$.

Lidocaína: es un agente anestésico local; el mecanismo de acción consiste en bloquear los canales de NA deteniendo la propagación del potencial de acción en las neuronas ${ }^{72}$.

La presentación es en parches al 5\% (Lidoderm); se utiliza en neuropatía periférica, aunque sólo está aprobado su uso en neuropatía post-herpética ${ }^{74}$.

La AAN indicó que hay débil evidencia para considerar a los parches de lidocaína al $5 \%$ en pacientes con NPD; si se utiliza, debería aplicarse un parche cada 12 hs dentro de un período de 24 hs $^{76}$.

Dicho parche puede producir lesiones o edema en la zona de aplicación y resultar en el aumento de las concentraciones de lidocaína en sangre por mayor absorción de la misma. Las reacciones adversas que se producen en el sitio de aplicación del parche incluyen: hematomas, despigmentación, dermatitis, pápulas, eritema, prurito y vesículas. Estas reacciones se alivian al retirar el parche.

Una de las ventajas del uso de lidocaína tópica es la asociación a otro tratamiento sistémico en cualquier momento6.

Amitriptilina local: la amitriptilina tópica ejercería efectos anestésicos periféricos por activación de receptores de adenosina y por inhibición de los canales de $\mathrm{Na}^{77}$.

De todas maneras, otros estudios han demostrado que la amitriptilina local no es efectiva sola ni asociada a otras drogas de uso local ${ }^{78,79,80}$.

. Toxina botulínica: ha sido probada para la neuralgia del trigémino y ha mostrado tener efecto anti-nociceptivo en el síndrome del túnel carpiano, sin mejoría electrofisiológica ${ }^{81,82}$.

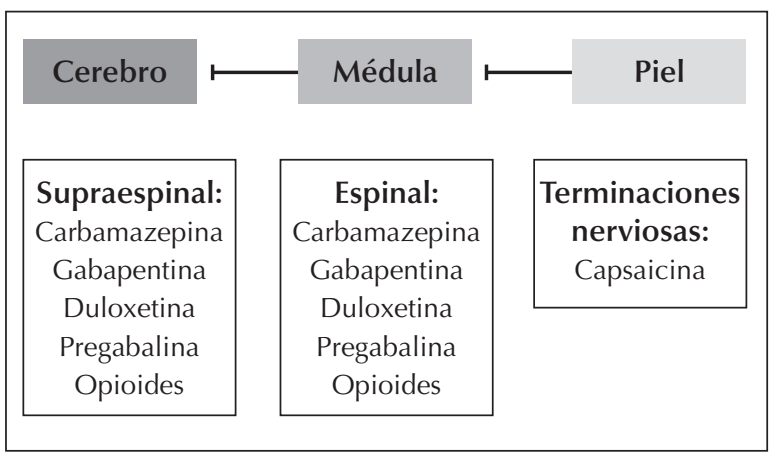

Gráfico 2: Sitios de acción en tratamiento actuales para el dolor neuropático ${ }^{98}$.

\begin{tabular}{|c|c|}
\hline \multicolumn{2}{|r|}{ Tratamiento farmacológico en NPD } \\
\hline Evidencia & Anticonvulsionantes \\
\hline Fuerte & Pregabalina (nivel A) \\
\hline Moderada & Gabapentina y valproato (nivel B) \\
\hline Insuficiente & Topiramato (nivel U) \\
\hline \multirow[t]{2}{*}{ Contexto clínico } & $\begin{array}{l}\text { Aunque el valproato es efectivo, es teratogénico, } \\
\text { por lo que debe evitarse en mujeres en edad } \\
\text { fértil. Debido a sus efectos adversos (incremento } \\
\text { de peso y empeoramiento del control glucémico) } \\
\text { no es droga de primera línea }\end{array}$ \\
\hline & Antidepresivos \\
\hline Fuerte & Duloxetina (nivel A) \\
\hline Moderada & $\begin{array}{l}\text { Amitriptilina, venlafaxina (nivel B). Los datos } \\
\text { son insuficientes para recomendar uno de estos } \\
\text { agentes sobre otros }\end{array}$ \\
\hline Débil & $\begin{array}{l}\text { Venlafaxina puede ser asociada a gabapentina } \\
\text { (nivel C) }\end{array}$ \\
\hline \multirow[t]{2}{*}{ Insuficiente } & $\begin{array}{l}\text { Desipramina, imipramina, fluoxetina, combina- } \\
\text { ción de nortriptilina y flufenazina }\end{array}$ \\
\hline & Opioides \\
\hline Moderada & $\begin{array}{l}\text { Dextrometorfano, morfina, tramadol, oxicodona } \\
\text { (nivel B) }\end{array}$ \\
\hline \multirow[t]{2}{*}{ Contexto clínico } & $\begin{array}{l}\text { El uso de opioides para el dolor no maligno } \\
\text { crónico ha ganado relevancia. } \\
\text { En la última década el uso de opioides se asocia } \\
\text { a nuevos síndromes dolorosos como cefalea de } \\
\text { rebote. El uso de tramadol y dextrometorfano aso- } \\
\text { ciados se relacionó a reacciones adversas como } \\
\text { sedación, náuseas y constipación. } \\
\text { El uso crónico de opioides produce tolerancia e } \\
\text { incremento frecuente de la dosis }\end{array}$ \\
\hline & Otros agentes farmacológicos \\
\hline Moderada & Capsaicina, spray de dinitrato de isosorbide (nivel B) \\
\hline Débil & Parches de lidocaína (nivel B) \\
\hline Contexto clínco & $\begin{array}{l}\text { Aunque la capsaicina ha sido utilizada en la dis- } \\
\text { minución del dolor en ensayos de NPD, muchos } \\
\text { pacientes son intolerantes a sus efectos adversos }\end{array}$ \\
\hline
\end{tabular}

Tabla 1: Guía basada en la evidencia (modificada). Asociación Americana de Neurología (AAN), $2011^{99}$. 


\begin{tabular}{|c|c|c|}
\hline & $\begin{array}{c}\text { Drogas recomendadas } \\
\text { y dosis }\end{array}$ & No recomendadas \\
\hline Nivel A & $\begin{array}{l}\text { Duloxetina } 60-120 \text { mg/día } \\
\text { Pregabalina } 300-600 \text { mg/día }\end{array}$ & \\
\hline Nivel B & $\begin{array}{l}\text { Gabapentina 900-3600 mg/día } \\
\text { Valproato de sodio 500-1200 } \\
\text { mg/día } \\
\text { Venlafaxina } 75-225 \mathrm{mg} / \text { día } \\
\text { Amitriptilina } 25-100 \mathrm{mg} / \mathrm{día} \\
\text { Dextrometorfano } 400 \mathrm{mg} / \mathrm{día} \\
\text { Sulfato de morfina titulado a } \\
120 \mathrm{mg} / \text { día } \\
\text { Tramadol } 210 \mathrm{mg} / \text { día } \\
\text { Oxicodona } 37 \mathrm{mg}-120 \mathrm{mg} / \text { día } \\
\text { Capsaicina 0,075\% } \\
\text { Electroestimulación, } \\
\text { estimulación nerviosa } \\
\text { percutánea por 3-4 semanales }\end{array}$ & $\begin{array}{l}\text { Oxacarbamacepina } \\
\text { Lamotrigina } \\
\text { Lecosamida } \\
\text { Clonidina } \\
\text { Pentoxifilina } \\
\text { Mexiletina } \\
\text { Tratamiento del campo } \\
\text { magnético } \\
\text { Terapia láser de baja } \\
\text { intensidad } \\
\text { Terapia Reiki }\end{array}$ \\
\hline
\end{tabular}

Tabla 2: Guía basada en la evidencia (modificada). Asociación Americana de Neurología (AAN), $2011^{99}$.

\section{Tratamiento no farmacológico}

En el tratamiento no farmacológico están incluidos los tratamientos alternativos complementarios, la psicoterapia y las medidas físicas. En la Tabla 3 se mencionan niveles de evidencia.

\section{- Tratamientos alternativos complementarios}

En algunas situaciones, el tratamiento farmacológico no es satisfactorio y en esos casos la terapéutica alternativa suele ser una opción a considerar. Una reciente revisión evaluó la evidencia de varios ensayos clínicos y metaanálisis de terapias complementarias y alternativas para el tratamiento del dolor neuropático y neurálgico. Dentro de los tratamientos alternativos pueden mencionarse: acupuntura, electroestimulación, medicina con hierbas, suplementos dietarios, magnetoterapia y sanación espiritual. La conclusión fue que la evidencia no es convincente para la mayoría de las modalidades de medicina alternativa y complementaria en la mejoría del dolor neuropático. Sin embargo la evidencia puede ser alentadora para nuevos estudios, para otras sustancias como extracto de cannabis, magnetoterapia, carnitina y electroestimulación ${ }^{83}$.

\section{- Psicoterapia}

El componente psicológico del dolor no debe desestimarse. Está justificado que los pacientes que presenten un dolor severo -particularmente aquellos con mal control metabólico, neuropatía dolorosa aguda o con síntomas dolorosos causa- dos por el tratamiento insulínico intensivo- sean derivados para realizar tratamiento psicológico ${ }^{84}$.

\section{- Medidas físicas}

En aquellos pacientes con pie neuropático doloroso, la temperatura puede estar aumentada por el shunt arterio-venoso. La inmersión en agua fría puede reducir el flujo del shunt y aliviar el dolor. La alodinia puede ser disminuida por el uso de pijama de seda o el hábito de dejar colgando las piernas fuera de la cama. En pacientes con síntomas dolorosos, el uso de calzado confortable es beneficioso para caminar sobre superficies irregulares ${ }^{84}$.

\begin{tabular}{|c|c|}
\hline \multicolumn{2}{|r|}{ Tratamiento no farmacológico en NPD } \\
\hline \multicolumn{2}{|c|}{$\begin{array}{l}\text { Electroestimulación, tratamiento de campo magnético } \\
\qquad y \text { otras intervenciones }\end{array}$} \\
\hline Moderada & $\begin{array}{l}\text { Electroestimulación (nivel B) } \\
\text { Magnetoterapia, tratamiento láser de baja intensidad, } \\
\text { terapia Reiki (nivel B) }\end{array}$ \\
\hline Insuficiente & Electroterapia solo o asociada a amitriptilina (nivel U) \\
\hline
\end{tabular}

Tabla 3: Guía basada en la evidencia (modificada). Asociación Americana de Neurología (AAN), 201199.

\section{Combinación e interacciones de drogas}

La respuesta a la monoterapia analgésica en el manejo del dolor en pacientes con PNDP es de aproximadamente $50 \%$, por lo tanto se requiere de la combinación farmacológica ante una respuesta parcial o en aquellos pacientes en quienes la titulación se ve afectada por los eventos adversos ${ }^{85}$.

Las combinaciones analgésicas adecuadas incluyen antidepresivos con anticonvulsivantes o cualquiera de éstos con opioides. Algunos pacientes pueden requerir una triple combinación de drogas $^{86,87}$.

Cuando se necesita combinar fármacos luego de utilizar la monoterapia, se debe considerar el mecanismo de acción y las interacciones medicamentosas. Por ejemplo, es importante evitar la administración de antidepresivos tricíclicos con ISRS o IRSN para prevenir el síndrome serotoninérgico, que se presenta con síntomas neurológicos centrales y autonómicos ${ }^{88}$.

Antes de iniciar una terapia para el dolor, debería revisarse una lista de potenciales interacciones fármacológicas en pacientes con comorbilidades. Las drogas que deberían incluirse son las estatinas, betabloqueantes, sulfonilureas, levotiroxina, warfarina, diuréticos de asa y drogas que intervienen en el metabolismo hepático a través del citocromo $\mathrm{P} 450^{89}$. 
En los Estados Unidos dos drogas han sido aprobadas por la FDA para utilizarse en el tratamiento del dolor neuropático: la pregabalina y duloxetina. En un metaanálisis reciente se comparó indirectamente duloxetina, con pregabalina y gabapentina para el tratamiento de la PNDP, y los resultados orientaron a que ambas drogas tienen comparable eficacia y tolerancia ${ }^{51}$. Algunos estudios han analizado los costos en salud en pacientes con PNDP tratados con pregabalina, duloxetina, u otras drogas de uso habitual. En general todas muestran resultados similares, con un buen perfil de costo-efectividad ${ }^{90,91,92}$.

El Panel de Consenso de Toronto en Neuropatía Diabética recomienda considerar como tratamiento de primera línea para la PND un agonista $\alpha 2 \delta$ (modulador de los canales de calcio), un inhibidor de la IRSN o un antidepresivo tricíclico. La elección del agente de primera línea se basa en las comorbilidades asociadas a la PNDP. Por ejemplo, personas con depresión comórbida se beneficiarían con duloxetina, que se puede utilizar para tratar tanto la depresión como el dolor neuropático con idéntica dosificación, mientras que la dosis eficaz para tratar el dolor neuropático con antidepresivos tricíclicos es en general insuficiente para el tratamiento de la depresión o la ansiedad. De igual manera, los antidepresivos tricíclicos, la duloxetina o la pregabalina serían una buena opción en pacientes con fibromialgia comórbida, mientras que en el caso de migraña crónica, una mejor opción sería la gabapentina o un antidepresivo tricíclico.

Las comorbilidades también contraindican un agente en particular, por ejemplo, los antidepresivos tricíclicos deben evitarse en personas con glaucoma, hipotensión ortostática, arritmias cardíacas o disuria. Las personas con pequeñas áreas de dolor localizadas podrían ser tratadas con agentes tópicos ${ }^{93}$.

En base a la evidencia proveniente de ensayos clínicos aleatorizados y controlados con placebo, la duloxetina debería ser el IRSN preferido y la pregabalina el agonista $\alpha 2 \delta$ de elección. Si el dolor no mejora con esta primera intervención, estos agentes podrían ser combinados, teniendo presentes las contraindicaciones de cada fármaco y las condiciones clínicas asociadas del paciente. De no controlarse con la terapia doble, se sugiere adicionar opioides como tramadol y oxicodona. El tratamiento escalonado con dosis habitualmente bien toleradas de cada fármaco es más eficaz y seguro que las dosis máximas de un solo fármaco administrado como monoterapia ${ }^{94}$.

La FDA sólo aprobó tres tratamientos para el alivio del dolor en pacientes con NPD: duloxetina (2004), pregabalina (2005) y tapentadol (2012). Sin embargo, se conocen otras drogas, como la gabapentina, venlafaxina, amitriptilina y oxicodona que son utilizadas comúnmente fuera de prospecto como terapia en neuropatía.

Existen diferente guías como la AAN, la de Mayo Clinic o las Guías NICE que tienen distintas prioridades en la utilización de las drogas ya mencionadas en esta revisión, de todos modos teniendo en cuenta las tres drogas aprobadas por la FDA, serían de primera línea, pudiéndose combinar con alguna otra de primera o segunda línea ${ }^{95,96,97}$.

Se debe tener en cuenta que al momento de iniciar un tratamiento sintomático es aconsejable asociar un tratamiento fisiopatológico, como es el ácido lipoico y la benfotiamina, drogas disponibles en nuestro medio.

\section{CONCLUSIONES}

La PNDP es una patología común y se asocia a un marcado empeoramiento de la calidad de vida de las personas que la padecen.

Aún no ha sido encontrada la terapéutica eficaz que pueda reparar el daño neurológico en forma integral, pero actualmente existen distintas alternativas terapéuticas que pueden, al menos, aliviar las manifestaciones de esta complicación.

\section{BIBLIOGRAFÍA}

1. Lee JH, Cox DJ, Mook DG, McCarty RC. Effect of hyperglycemia on pain threshold in alloxan-diabetic rats. Pain. 1990; 40(1):105-107.

2. Moss SE, et ál. WESDR. Arch. Intern. Med. 1992; 152: 610-616.

3. DCCT Research Group. N. Engl. J. of Med. 1993; 329: 977-986.

4. OhkuboY, et ál. Diabetes Res. Clin. Pract. 1995; 28: 103-117.

5. Turner RC, Holman RR, Cull CA, et al. Intensive blood-glucose control with sulphonylureas or insulin compared with conventional treatment and risk of complications in patients with type 2 diabetes (UKPDS 33). Lancet. 1998: 352; (9131): 837-853.

6. Tammy JL, Blake CR, Vincent S, et al.Treating diabetic peripheral neuropathic pain. Saint Louis University Family Medicine Residency Program, Belleville, Illinois. American Family Physician. 2010; 82, (2): 151-158.

7. Brownlee M. Metabolism. 2000; 49 (2 sppl 1): 9-13.

8. Greene DA, et ál. N. Engl. J. Med. 1987; 316:599-606.

9. Javed S, Malik R.Therapeutic Advances in Chronic Disease 2015; 6(1): 15-28.

10. Albers JW, Herman WH, PopBusui R, et al. Effect of prior intensive insulin treatment during the Diabetes Control and Complications Trial (DCCT) on peripheral neuropathy in type 1 diabetes during the Epidemiology of Diabetes Interventions and Complications (EDIC) Study. Diabetes Care 2010; 33, (5): 1090-1096. 
11. PopBusui R, Low PA, Waberski BH, et al. Effects of prior intensive insulin therapy on cardiac autonomic nervous system function in type 1 diabetes mellitus. The Diabetes Control and Complications Trial/Epidemiology of Diabetes Interventions and Complications Study (DCCT/EDIC). Circulation 2009; 119, (22): 2886-2893.

12. Patel A, MacMahon S, Chalmers J, et al. Intensive blood glucose control and vascular outcomes in patients with type 2 diabetes. ADVANCE Collaborative Group. N. Engl. J. Med. 2008; 358 (24): 2560-2572.

13. Duckworth W, Abraira C, MoritzT, et al. Glucose control and vascular complications in veterans type 2 diabetes. VADT Investigators. N. Eng. J. Med 2009; 360 (2): 129-139.

14. Boussageon R, Bejan-Angoulvant $T$, Saadatian-Elahi M, et al. Effect of intensive glucose lowering treatment on all cause mortality, cardiovascular death, and microvascular events in type 2 diabetes: metaanalysis of randomised controlled trials. BMJ 2011; 343: d4169.

15. Callaghan B, Little A, Feldman E, et al. Enhanced glucose control for preventing and treating diabetic neuropathy. Cochrane Database Syst. Rev 6: CD007543.

16. Sociedad Argentina de Diabetes. Guías de práctica clínica para el manejo de la diabetes tipo 1. Buenos Aires, 2006.

17. Ziegler D. Treatment of diabetic neuropathy and neuropathy pain: How far have we come? Diabetes Care 31 (Suppl. 2): S255261, 2008.

18. Fioretto P, Steffes M, Sutherland D, et al. Reversal of lesions of diabetic nephropathy after pancreas transplantation. N. Engl. Med. 1998; 339: 69-75.

19. Vincent A, Callaghan B, Smith A, et al. Diabetic neuropathy: ceIlular mechanisms as therapeutic targets. Nat. Rev. Neurol. 2011; 7: $573-583$.

20. Ametov AS, Barinov A, Dyck PJ, et al. The sensory symptoms of diabetic polyneuropathy are improved with alpha-lipoic acid: the SYDNEY trial. Diabetes Care 2003; 26: 770-776.

21. Ruhnau KJ, Meissner HP, Finn R, et al. Effects of 3-week oral treatment with the antioxidant thioctic acid (alpha-lipoic acid) in symptomatic diabetic polyneuropathy. Diabet. Med. 1999; 16 (12): 1040-1043.

22. Ziegler D, Hanefeld M, Ruhnau KJ, et al. Treatment of symptomatic diabetic peripheral neuropathy with the anti-oxidant alpha-lipoic acid. A 3-week multicentre randomized controlled trial (ALADIN Study). Diabetologia 1995; 38:1425-1433.

23. Reljanovic M, Reichel G, Rett K, et al. Treatment of diabetic polyneuropathy with the antioxidant thioctic acid (alpha-lipoic acid): a two year multicenter randomized double-blind placebo-controlled trial (ALADIN II). Alpha lipoic acid in diabetic neuropathy free radic. Res. 199931 (3): 171-179.

24. Ziegler D, et al. Oral treatment with alfa lipoic acid improves symptomatic diabetic polyneuropathy: the SYDNEY 2 trial. Diabetes Care 2006, 29:2365-70.

25. Ziegler D, et al. Antioxidant treatment with alpha lipoic acid in diabetic polyneuropathy. A 4-yearsrandomiseddoublé-blinded trial (NATHAN 1 Study). Diabetologia 2007, 50; Suppl 1, S63.

26. Ziegler D, Nowak H, Kempler P, et al. Treatment of symptomatic diabetic polyneuropathy with the antioxidant alpha-lipoic acid: a meta-analysis. Diabet Med. 2004 21: 114-121.

27. Ziegler D, et al. Treatment of symptomatic polyneuropathy with actovegin in type 2 diabetic patients. Diabetes Care 2009; 32:1479-1484.

28. Ziegler D. Current concepts in the management of diabetic polyneuropathy. Current Diabetes Reviews 2011; 7: 208220.
29. Hammes H, Du X, Edelstein D, et al. Benfotiamine blocks three major pathways of hyperglycemic damage and prevents experimental diabetic retinopathy. Nat. Med. 2003 9: 294-299.

30. Stracke H, Gaus W, Achenbach U, et al. Benfotiamine in Diabetic Polyneuropathy (BENDIP): Results of a randomised, double blind, placebo-controlled Clinical Study. Exp. Clin. Endocrinol. Diabetes 2008; 116: 600-605.

31. Boulton A, Kempler P, Ametov A, Ziegler D. Whither pathogenetic treatments for diabetic polyneuropathy. Diabetes Metab. Res. Rev. 2013; 29: 327-333.

32. Goto $Y$, Hotta $N$, Shigeta $Y$, et al. Effects of aldose reductase inhibitor, epalrestat, on diabetic neuropathy, clínic benefits and indications for the drug assessed from the results of a placebocontrolled double blind study. Biomed. Pharmacother 1995; 49: 269-277.

33. Hotta N, AkanumaY, Kawamori R, et al. Long term clinical effects of epalrestat, and aldose reductase inhibitor, on diabetic peripheral neuropathy: the 3-year, multicenter, comparative aldose reductase inhibitor-diabetes complications trial. Diabetes Care 2006; 29:1538-1544.

34. Keen H, Payan J, Allawi J, et al. Treatment of diabetic neuropathy with linolenic acid. Diabetes Care 1993;16:8-15.

35. Scafidi S, Fiskum G, Lindauer SL, et al. Metabolism of acetil-carnitine for energy and neurotransmiter synthesis in the immature rat brain. J. Neurochem. 2010; 114: 820-831.

36. Evans JD, Jacobs TF, Evans EW. Role of acetyl-L-carnitine in the treatment of diabetic peripheral neuropathy. Ann. Pharmacoter. 2008; 42: 1686-1691.

37. Finnerup NB, Otto $\mathrm{M}, \mathrm{McQuay} \mathrm{HJ}$, et al. Algorithm for neuropathic pain treatment: an evidence based proposal. Pain 2005; 118: 289-305.

38. Max MB, Lynch SA, Muir J, et al. Effects of desipramine, amitriptyline, and fluoxetine on pain in diabetic neuropathy. N. Engl. J. Med. 1992; 326: 1250-1256.

39. Ulugol A, Karadag HC, Tamer M. Involvement of adenosine in the antiallody nice effect of amitriptyline in streptozotocin-induced diabetic rats. Neurosci. Lett. 2002; 328: 129-132.

40. Saarto T, Wiffen PJ. Antidepressants for neuropathic pain. Cochrane Database Syst. Rev. 2007; 4: CD005454.

41. US Food and Drug Administration. Medication guide: Pamelor, http://www.fda.gov/downloads/Drugs/DrugSafety/ucm088671. pdf (accessed 27 April 2014).

42. Moulin DE, Clark AJ, Gilron I, et al. Pharmacological management of chronic neuropathic pain-consensus statementand guidelines from the Canadian Pain Society. Pain Res. Manag. 2007; 12: 13-21.

43. Gilron I, Bailey JM, Tu D, et al. Nortriptyline and gabapentin, alone and in combination for neuropathic pain: a double-blind, randomised controlled crossover trial. Lancet 2009; 374: 1252-1261.

44. Morello CM, Leckband SG, Stoner CP, et al. Randomized doubleblind study comparing the efficacy of gabapentin with amitriptyline on diabetic peripheral neuropathy pain. Arch. Intern. Med. 1999; 159: 1931-1937.

45. Sima AA, Calvani M, Mehra M, et al. Acetyl-I-carnitine improves pain, nerve regeneration, and vibratory perception in patients with chronic diabetic neuropathy: an analysis of two randomized placebo-controlled trials. Diabetes Care 2005; 28: 89-94.

46. National Institute for Health and Clinical Excellence. Neuropathic pain-pharmacological management: the farmacological management of neuropathic pain in adults in non-speciallist settings. 2013.

47. Vinik A, et al. Evidence based management of diabetes. 2012 Chapter 19, 348-349. 
48. Vinik A, Caselli C, Nevoret ML. Endotext. Diabetic neuropathy. Last Update: august 2015.

49. Kajdasz DK, lyengar S, Desaiah D, et al. Duloxetine for the management of diabetic peripheral neuropathic pain: evidence-based findings from post hoc analysis of three multicenter, randomized, double-blind, placebo-controlled, parallel-group studies. Clin. Ther. 2007; 29 Suppl: 2536-2546.

50. Wernicke JF, Pritchett YL, D'Souza DN, et al. A randomized controlled trial of duloxetine in diabetic peripheral neuropathic pain. Neurology 2006; 67: 1411-1420.

51. Quilici S, Chancellor J, Lothgren M, et al. Meta-analysis of duloxetine vs pregabalin and gabapentin in the treatment of diabetic peripheral neuropathic pain. BMC Neurology 2009; 9: 6. doi: 10.1186/1471-2377-9-6.

52. Montfort EG, Witte AP, Ward K. Neuropathic pain: a review of diabetic neuropathy. US Pharm 2010; 35: HS8-HS15.

53. Hardy T, Sachson R, Shen S, et al. Does treatment with duloxetine for neuropathic pain impact glycemic control? Diabetes Care 2007; 30:21-26.

54. Sansone RA, Sansone LA. Pain, pain, go away: antidepressants and pain management. Psychiatry (Edgmont ) 2008; 5: 16-19.

55. Effexor XR (venlafaxine extended release) prescribing information. Philadelphia,Pennsylvania: Wyeth Pharmaceuticals, Inc.; February 2015.

56. La Roche SM, Helmers SL. The new antiepileptic drugs: scientific review. JAMA 2004; 291: 605-14. 128,132.

57. Lesser $H$, Sharma $U$, LaMoreaux $L$, et al. Pregabalin relieves symptoms of painfuldiabetic neuropathy: a randomized controIled trial. Neurology 2004; 63:2104-10.

58. Richter RW, Portenoy R, Sharma U, et al. Relief of painful diabetic peripheral neuropathy with pregabalin: a randomized, placebo-controlled trial. J. Pain 2005; 6: 253-60.

59. Moulin DE, Boulanger A, Clark AJ, et al. Pharmacological management of chronic neuropathic pain: revised consensus statement from the Canadian Pain Society. Pain Res. Manag. 2014; 19(6):328-335.

60. Freeman R, Durso-Decruz E, Emir B. Efficacy, safety, and tolerability of pregabalin treatment for painful diabetic peripheral neuropathy: findings from seven randomized, controlled trials across a range of doses. Diabetes Care 2008; 31:1448-54.

61. Backonja M, Beydoun A, Edwards KR, et al. Gabapentin for the symptomatic treatment of painful neuropathy in patients with diabetes mellitus: a randomized controlled trial. JAMA. 1998;280:1831-1836.

62. DeToledo JC, Toledo C, De Cerce J, et al. Changes in body weight with chronic, high-dose gabapentin therapy. Ther. Drug Monit. 1997;19:394-396.

63. Gilron I, Bailey JM, Tu D, et al. Morphine, gabapentin, or their combination for neuropathic pain. N. Engl. J. Med. 2005; 352:1324-1334.

64. Schreiber AK, Nones $\mathrm{CF}$, Reis RC, et al. Diabetic neuropathic pain: Physiopathology and treatment. World J. Diabetes 2015 April 15; 6(3): 432-444.

65. Kalso E. Sodium channel blockers in neuropathic pain. Curr. Pharm. Des. 2005; 11:3005-3011.

66. Vinik Al. Diabetic neuropathies: endpoints in clinical research studies. In: Le Roith D, Vinik Al, editors. Controversies in treating diabetes: clinical and research aspects (Contemporary Endocrinology). Totowa, NJ: Human Press; 2008:135-156.

67. Eisenberg E, McNicol E, Carr DB. Opioids for neuropathic pain. Cochrane Database Syst. Rev. 2006; (3):CD006146.
68. Watson $\mathrm{CP}$, Moulin D, Watt-Watson J, et al. Controlled-release oxycodone relieves neuropathic pain: a randomized controlled trial in painful diabetic neuropathy. Pain. 2003;105(1-2):71-78.

69. Sang CN. NMDA-receptor antagonists in neuropathic pain: experimental methods to clinical trials. J. Pain Symptom Manage 2000; 19:S21-S25.

70. Schwartz S, Etropolski M, Shapiro DY, et al. Safety and efficacy of tapentadol in patients with painful diabetic peripheral neuropathy: results of a randomized-withdrawal, placebo-controlled trial. Curr. Med. Res. Opin. 2011; 27:151-162.

71. Harati $Y$, Gooch $C$, Swenson M, et al. Double-blind randomized trial of tramadol for the treatment of the pain of diabetic neuropathy. Neurology 1998; 50:1842-1846.

72. Cummins TR. Setting up for the block: the mechanism underlying lidocaine's use-dependent inhibition of sodium channels. J. Physiol. 2007; 582 (Pt 1): 11.

73. Rosenberg CJ, Watson JC. Treatment of painful diabetic peripheral neuropathy. Prosthetics and Orthotics International 2015, Vol. 39 (1) 17- 28.

74. Cohen K, Shinkazh N, Frank J, et al. Pharmacological treatment of diabetic peripheral neuropathy. $P \& T^{\oplus}$, June 2015 , Vol. $40 \mathrm{~N}^{\circ} 6$.

75. American Academy of Neurology. AAN summary of evidencebased guidelines for clinicians: treatment of painful diabetic neuropathy. 2011. Availableat: https://www.aan.com/Guidelines/ home/GetGuidelineContent/480. Accessed March 13, 2015.

76. Montfort EG, Witte AP, Ward K. Neuropathic pain: a review of diabetic neuropathy. US Pharm 2010; 35:HS8-HS15.

77. Flores MP, de Castro APCR, Nascimento JDS. Topical analgesics. Rev. Bras. Anestesiol. 2012; 62:244-252.

78. Ho K, Huh BK, White WD, et al. Topical amitriptyline versus lidocaine in the treatment of neuropathic pain. Clin. J. Pain 2008;24:51-55.

79. Lynch ME, Clark AJ, Sawynok J, et al. Topical $2 \%$ amitriptyline and $1 \%$ ketamine in neuropathic pain syndromes: a randomized, double-blind, placebo-controlled trial. Anesthesiology 2005; 103:140-146.

80. Estebe JP, Myers RR. Amitriptyline neurotoxicity: dose-related pathology after topical application to rat sciatic nerve. Anesthesiology 2004; 100:1519-1525.

81. Piovesan EJ, Teive HG, Kowacs PA, et al. An open study of botulinum. A toxin treatment of trigeminal neuralgia. Neurology 2005; 65:1306-1308.

82. Tsai CP, Liu CY, Lin KP, et al. Efficacy of botulinum toxin type a in the relief of Carpal tunnel syndrome: A preliminary experience. Clin. Drug Investig. 2006; 26:511-515.

83. Pittler MH, Ernst E. Complementary therapies for neuropathic and neuralgic pain: systematic review. Clin. J. Pain 2008; 24:731-733.

84. Tesfaye S. Painful diabetic neuropathy. Aetiology and non pharmacological treatment. In: Veves A, editor. Clinical management of diabetic neuropathy. Totowa (NJ): Human Press; 1998. p. 369-86.

85. Gilron I, Bailey JM, Tu D, Holden RR, Jackson AC, Houlden RL. Nortriptyline and gabapentin, alone and in combination for neuropathic pain: a double-blind, randomised controlled crossover trial. Lancet 2009; 374:1252-1261.

86. Tesfaye S, Vileikyte L, Rayman G, et al. Painful diabetic peripheral neuropathy: consensus recommendations on diagnosis, assessment and management. Diabetes Metab. Res. Rev. 2011;27:629-638.

87. Lexi-Comp [online reference library]. Hudson, Ohio: American Pharmaceu-tical Association; 2009. Updated daily. http://online. lexi.com (subscription required). Accessed October 27, 2009. 
88. Boyer EW, Shannon M. The serotonin syndrome [published corrections appear in N Engl J Med. 2009;361(17):1714 and N. Engl. J. Med. 2007; 356:(23):2437]. N. Engl. J. Med. 2005; 352(11):1112-1120.

89. Gore M, Sadosky A, Leslie D, et al. Selecting an appropriate medication for treating neuropathic pain in patients with diabetes: a study using the U.K. and Germany Mediplus databases. Pain Pract. 2008; 8(4):253-262.

90. Burke JP, Sanchez RJ, Joshi AV, et al. Health care costs in patients with painful diabetic peripheral neuropathy prescribed pregabalin or duloxetine. Pain Pract. 2012; 12:209-218.

91. De Salas-Cansado M, Perez C, Saldana MT, et al. An economic evaluation of pregabalin versus usual care in the management of community-treated patients with refractory painful diabetic peripheral neuropathy in primary care settings. Prim Care Diabetes. Epub May 15, 2012.

92. Bellows BK, Dahal A, JiaoT, Biskupiak J. A cost-utility analysis of pregabalin versus duloxetine for the treatment of painful diabetic neuropathy. J. Pain Palliat Care Pharmacother. 2012; 26:153-164.

93. Tesfaye S, Vileikyte L, Rayman G, et al. Painful diabetic peripheral neuropathy: consensus recommendations on diagnosis, assessment and management. Diabetes Metab. Res. Rev. 2011; 27:629-638.
94. Vinik A. The approach to the management of the patient with neuropathic pain. J. Clin. Endocrinol. Metab. 2010; 95:4802-4811.

95. Bril V, England J, Franklin GM, et al. Evidence based guideline: treatment of painful diabetic neuropathy: report of the American Academy of Neurology, the American Association of Neuromuscular and Electrodiagnostic Medicine, and the American Academy of Physical Medicine and Rehabilitation. Neurology 2011; 76:1758-1765.

96. Argoff C, Backonja M, Belgrade M, et al. Consensus guidelines: treatment and planning options: diabetic peripheral neuropathic pain. Mayo Clin Proc. 2006; 81 (suppl):S12-S25.

97. Tan T, Barry P, Reken S, et al. Pharmacological management of neuropathic pain in non-specialist settings: summary of NICE guidance. BMJ 2010; 340:c1079.

98. Javed S, Petropoulos LN, Alam U, et al. Treatment of painful diabetic neuropathy. Ther. Adv. Chronic. Dis. 2015, Vol. 6 (1) 15-28.

99. Summary of evidence-based Guideline for Clinicians treatment of painful diabetic neuropathy. American Academy of Neurology (AAN) guideline update regarding pharmacologic and nonpharmacologic treatment of painful diabetic neuropathy (PDN) 2011.

\section{Fe de erratas}

En el Vol. 49, N Especial XVI "Jornadas de Graduados en Diabetes", de Noviembre de 2015, en la página 22, Tabla 1, donde figuraba:

\begin{tabular}{|c|c|c|}
\hline & $\begin{array}{l}\text { Accu Chek } \\
\text { Spirit Combo }\end{array}$ & $\begin{array}{l}\text { Paradigm MMT } \\
554 / 754\end{array}$ \\
\hline $\begin{array}{l}\text { Catéter-agujas } \\
\text { Tipo de cánula: } \\
\text { — Teflón } \\
\Delta \text { Metálica } \\
\diamond \text { Inserción en ángulo } \\
\text { O Insertador }\end{array}$ & $\begin{array}{l}\text { Tenderlink } 13 \mathrm{~mm}, 17 \mathrm{~mm} \boldsymbol{\square} \\
\text { Flexlink } 8 \mathrm{~mm} \text {, } \\
10 \mathrm{~mm} \mathbf{0} \\
\text { Rapid-D-Link } 6 \mathrm{~mm}, 8 \mathrm{~mm} \boldsymbol{\Delta} \\
\text { Link Assist para Flexlink }\end{array}$ & $\begin{array}{l}\text { Silhouette } 13 \mathrm{~mm}, 17 \mathrm{~mm} \mathbf{} \\
\text { Quick-set } 6 \mathrm{~mm}, 9 \mathrm{~mm} \mathbf{0} \\
\text { Soft-set } 6 \mathrm{~mm} \text {, } \\
9 \mathrm{~mm} \mathbf{0}\end{array}$ \\
\hline
\end{tabular}

Debía figurar:

\begin{tabular}{|c|c|c|}
\hline & $\begin{array}{l}\text { Accu Chek } \\
\text { Spirit Combo }\end{array}$ & $\begin{array}{l}\text { Paradigm MMT } \\
554 / 754\end{array}$ \\
\hline $\begin{array}{l}\text { Catéter-agujas } \\
\text { Tipo de cánula: } \\
\text { - Teflón } \\
\text { A Metálica } \\
\searrow \text { Inserción en ángulo } \\
\text { - Insertador }\end{array}$ & $\begin{array}{l}\text { Tenderlink } 13 \mathrm{~mm}, 17 \mathrm{~mm} \\
\text { Flexlink } 6 \mathrm{~mm}-10 \mathrm{~mm} / \\
\text { Rapid D Link } 6 \mathrm{~mm}-10 \mathrm{~mm} \\
\text { Link Assist para aguja Flexlink }\end{array}$ & $\begin{array}{l}\text { Silhouette } 13 \mathrm{~mm}, 17 \mathrm{~mm} \mathbf{} \\
\text { Quick-set } 6 \mathrm{~mm}, 9 \mathrm{~mm} \mathbf{0} \\
\text { Soft-set } 6 \mathrm{~mm} \text {, } \\
9 \mathrm{~mm} \mathbf{0} \\
\text { Quick serter } \\
\text { Sil Serter (para silhouette) }\end{array}$ \\
\hline
\end{tabular}

